\title{
Boundary Integral Equations in the Frequency Domain for Interface Cracks under Impact Loading
}

\author{
Oleksandr Menshykov ${ }^{1, *}$ and Marina Menshykova ${ }^{1,2}$ \\ ${ }^{1}$ Centre for Micro and Nanomechanics (CEMINACS), School of Engineering, College of Physical Sciences, University of \\ Aberdeen, AB24 3UE, Scotland, UK \\ 2 Ziebel UK Ltd, Broadfold Road, Bridge of Don, Aberdeen, AB23 8EE, Scotland, UK
}

\begin{abstract}
In the study interface cracks between two dissimilar homogeneous isotropic half-spaces undergoing impact loading are considered. The problem is solved using the boundary integral equations in the frequency domain. The distributions of the displacements and tractions at the bonding interface and surfaces of the cracks are obtained and analyzed. The stress intensity factors (opening and shear modes) are computed for different load conditions and various properties of the bimaterial. The results are compared with the ones obtained in the time domain.
\end{abstract}

(c) 2012 Wiley-VCH Verlag GmbH \& Co. KGaA, Weinheim

\section{Introduction}

In order to meet the requirements of modern design and service conditions, mechanical systems and structures are subject to further and further increases in both the magnitude and frequency of loading. Subsequent research in the field of materials science has yielded new high-tech materials, in particular composites, which have enabled significant improvements in the strength and stiffness of these systems and structures to be made. However it has been apparent from the early days that engineering materials are inherently flawed, as they contain irregularly dispersed microdefects such as cracks, voids and delaminations, which may originate from various reasons. The presence of such flaws may greatly and detrimentally affect the stiffness and strength, and overall integrity of materials, leading to spontaneous fracture under the loading with unexpectedly small magnitude [1].

\section{Problem statement and results}

Consider a linear crack located at the bimaterial interface between two isotropic and linear elastic half-spaces under action of the transient wave. In the absence of body forces, the stress-strain state of both domains is defined by the dynamic Lamé equations. We assume that there are no initial displacements of the points of the material and the continuity conditions for displacements and stresses are satisfied at the bonding interface. In addition, the Sommerfeld radiation-type condition, which ensures a finite elastic energy of the infinite body, is imposed at the infinity on the vector of displacements.

The external dynamic load has been represented by the Fourier exponential series. The system of boundary integral equations for the displacements and tractions at the bonding interface and the crack surface for the case considered was derived using Somigliana dynamic identity (analogous to the system derived in [2,3]. The obtained system of integral equations was solved numerically by the boundary elements method.

As a model numerical example we considered an incident pulse of the unit amplitude propagating in the normal direction to the interface of the linear crack with the length of $2 a$. For the validation of the results the properties of both half-spaces were set identical, the Young modulus $E=200 G P a$, Poisson's ratio $\nu=0.25$, and the density of the material $\rho=7800 \mathrm{~kg} / \mathrm{m}^{3}$.

The dynamic stress intensity factors (opening mode) obtained for the Heaviside pulse $H(t)$ and the triangular pulse of the form $\frac{t}{t^{*}}\left(H(t)-H\left(t-t^{*}\right)\right)+\left(2-\frac{t}{t^{*}}\right)\left(H\left(t-t^{*}\right)-H\left(t-2 t^{*}\right)\right)$ and normalized by the corresponding static value are given in Figures 1 and 2, where circles refer to the numerical solution obtained in the time domain by Zhang and Wuensche (results have been kindly provided to the authors by the German side) and the dashed line refers to the analytical solution obtained for the Heaviside pulse by Baker [4]. The obtained results are in very good agreement with the model ones which clearly proves the applicability of the method proposed.

The results for cracks between dissimilar materials have also been obtained and analyzed for different properties of materials and various directions and forms of the pulse, including trapezoidal and half-sinusoidal ones.

Note that in reality the opposite crack faces under dynamic loading would interact with each other significantly affecting the stress-strain distribution at the vicinity of the crack. Considering this interaction will be the natural next stage of the research.

\footnotetext{
* Corresponding author: e-mail o.menshykov@abdn.ac.uk, phone +44 1224 273 326, fax +44 1224272519
} 


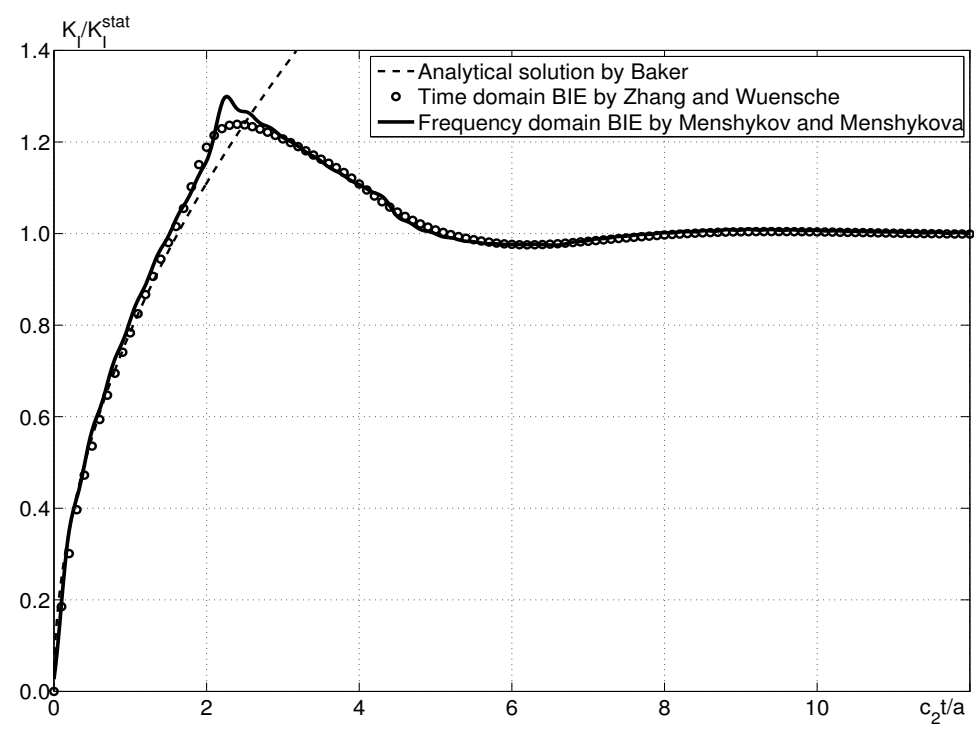

Fig. 1: Stress intensity factor (opening mode) vs. time for the Heaviside pulse

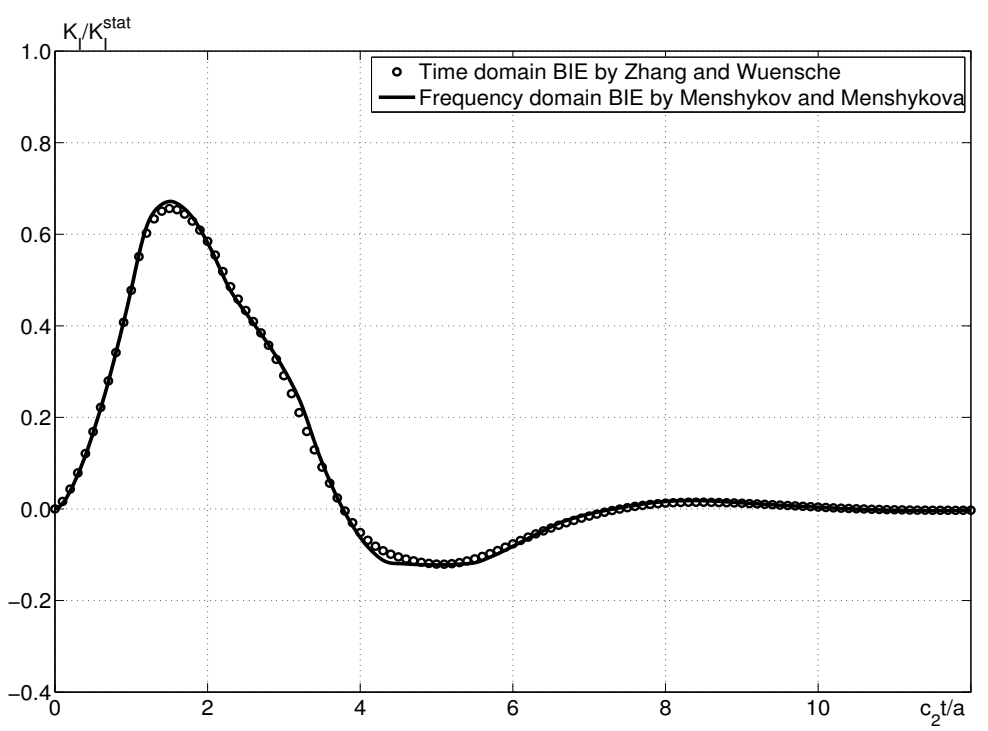

Fig. 2: Stress intensity factor (opening mode) vs. time for the triangular pulse, $t^{*}=1.12$

Acknowledgements The first author is thankful to the German Academic Exchange Service (DAAD) and Advanced Research Collaboration (ARC) Programme (funded by the British Council) for the financial support. The authors are very grateful to Prof. Chuanzeng Zhang and Dr. Michael Wuensche (University of Siegen, Germany) for the valuable suggestions and numerical results provided for the validation, and to Mr. Mark Lawrence (undergraduate MEng student, School of Engineering, University of Aberdeen) for the helpful discussions.

\section{References}

[1] Ch. Zhang and D. Gross, On Wave Propagation in Elastic Solids with Cracks (Computational Mechanics Publications, 1998).

[2] O. V. Menshykov, I. A. Guz and V. A. Menshykov, Phil Trans R Soc 366, 1835-1839 (2008).

[3] O. V. Menshykov, V. A. Menshykov and I. A. Guz, Eng Anal Bound Elem 33(3), 294-301 (2009).

[4] B. R. Baker, J Appl Mech 29, 449-454 (1962). 